\title{
Real models: The limits of behavioural evidence for understanding the ANS
}

\author{
Denitza Dramkin (ddramkin@psych.ubc.ca) \\ Darko Odic (darko.odic@psych.ubc.ca)
}

Department of Psychology, University of British Columbia, Vancouver, BC, V6T 1Z4, Canada

\begin{abstract}
Clarke and Beck use behavioural evidence to argue that (1) approximate ratio computations are sufficient for claiming that the approximate number system (ANS) represents the rationals, and (2) the ANS does not represent the reals. We argue that pure behaviour is a poor litmus test for this problem, and that we should trust the psychophysical models that place ANS representations within the reals.
\end{abstract}

Clarke and Beck (C\&B) ask what the approximate number system (ANS) represents, but an equally important question is what approach should we use to answer this question? C\&B put behavioural evidence above all other arguing that (1) behaviourally attested ratio computations are sufficient for the claim that the ANS represents the rationals, and (2) the absence of evidence that the ANS can compute $\pi$ or $\sqrt{2}$ shows that it (probably) cannot represent the reals.

However, there are meaningful challenges with both these arguments.

First, C\&B rely primarily on research showing that observers can reason about ratios of approximate number representations. For example, observers are not only able to judge that the side with 20 dots appears more numerous than the side with 10, but also that one side has (approximately) twice as many dots as the other (Jacob, Vallentin, \& Nieder, 2012). However, this behavioural finding does not guarantee that ANS representations, per se, are rational. It remains entirely possible that whole-numbered ANS representations merely serve as inputs for ratio computations, which are outputted to an entirely separate, domain-general ratio scale (Luce, Steingrimsson, \& Narens, 2010; Matthews \& Chesney, 2015). Such a scale could represent the ratio of not only two ANS representations, but also two lengths of lines, two sizes of objects, or even - in cross-modal matching tasks - be used to make "a sound three times as loud as [a] light is bright" (de Hevia, Vanderslice, \& Spelke, 2012; Ellermeier, Kattner, \& Raum, 2021).

$\mathrm{C} \& \mathrm{~B}$ are aware of this possibility, but puzzlingly note that it matters little for their arguments, as both the ANS and something like a ratio processing system (RPS) follow Weber's law. Yet adherence to Weber's law does little to unify these competing accounts. Noisy ANS representations of whole numbers could easily show Weber's law in ratio computations: An observer seeing a collection of 20 versus 10 dots could input a noisy signal of 20:10,18:12, or even 22:9 dots into a ratio operation and outputted to an RPS. This system would then inherit Weber's law without sharing any other properties with the ANS. In fact, despite C\&B suggesting that ratio computations are likely encapsulated within the ANS, there is good reason to suspect that these computations can exist entirely separately from it. A domain-general ratio scale easily explains how cross-modal matching tasks are accomplished (e.g., readily matching the ratio between sets of dots to two lines; Bonn \& Cantlon, 2017). Moreover, individual differences in the ANS do not correlate with ratio operations in other perceptual domains (e.g., length and area; Dramkin \& Odic, 2020; Odic, 2018), suggesting that the ANS is not the bottleneck for ratio computation. But, if ratio computations "live" in an entirely separate system, then $\mathrm{C} \& \mathrm{~B}$ have only presented behavioural evidence that this system represents the rationals, not the ANS, itself.

A second challenge is in the argument that the ANS does not represent the reals. Both $\mathrm{C} \& \mathrm{~B}$, as well as Laurence and Margolis (2005), nicely frame this as the question of the "grain of representations": what is the minimal unit on the scale of the ANS? Both sets of authors argue that 
until we find behavioural evidence that, for example, the mind represents $\pi$ or $\sqrt{ } 2$, we should not claim that the ANS is a real-numbered system, harkening to classic debates surrounding perceptual grain sizes. Fechner (1887) famously proposed that the unit of any perceptual dimension is the point at which observers fail to notice an objective increase in that quantity (i.e., the just noticeable difference; JND). But many psychophysicists, including Stevens (1961, 1957), argue that such behavioural "scales of confusability" tell us little about the units of perception because performance factors always interfere with true competency. Consider the case of absolute thresholds of light detection. In ideal situations, even a single photon of light can excite a rod cell in the retina. But observers don't always detect this, likely because of biological noise in the optical nerve or because of a balance that vision has to make between accepting signal versus rejecting noise (Ala-Laurila \& Rieke, 2014; Barlow, 1956; Rieke \& Baylor, 1998). As a result, pure behavioural signatures are a poor indicator of a perceptual system's true capabilities, which is why researchers studying absolute thresholds use models of performance coupled with potential sources of noise in the signal, biology of the eye, and the observer's decision making to understand absolute thresholds (Field, Sampath, \& Rieke, 2005). In the same way, pure behavioural data are too poor (from performance limitations) to tell us whether observers can represent the infinity of $\pi$ versus merely 355/113.

What, then, can we conclude about ANS representations if we go beyond pure behaviour? Much like the case of light perception, the best available approach is to rely on models of the ANS, including signal detection approaches that model perceptual signals as highly continuous and in the domain of the reals (Piazza, Izard, Pinel, Le Bihan, \& Dehaene, 2004; Pica, Lemer, Izard, \& Dehaene, 2004). Models fill the gaps between behavioural data, especially at the limits of behaviour where performance factors occlude true competency. Thus, the onus on proving that the grain size of the ANS is at the level of rationals must fall on demonstrating where current models of the reals break down. In other words, what does the model predict that is explicitly tested and not shown in behaviour (given all appropriate controls, sources of noise, etc.)? Ultimately, without an alternative to challenge the orthodox model of the ANS proper, we can only commit to the reals.

Although we agree with $\mathrm{C} \& \mathrm{~B}$ that many nuances of ANS representations have not yet been derived nor tested, determining what the ANS represents will rest on settling what evidence is sufficient. As we've argued, the challenge with relying on behaviour as the sole source of evidence is that it is neither sufficient to tell us that the ANS represents the rationals nor that it doesn't represent the reals. Instead, we should rely on psychophysical models that can go beyond the limits of behaviour to get at the true capabilities of perception and thought.

Financial support. This work is supported by a Jacobs Fellowship and a Natural Sciences and Engineering Discovery Grant awarded to D.O.

Conflict of interest. The authors declare no conflict of interest.

\section{References}

Ala-Laurila, P., \& Rieke, F. (2014). Coincidence detection of singlephoton responses in the inner retina at the sensitivity limit of vision. Current Biology 24(24):2888-2898. doi: 10.1016/j.cub.2014.10.028.

Barlow, H. B. (1956). Retinal noise and absolute threshold. Journal of the Optical Society of America 46(8):634-639. doi: 10.1364/JOSA.46.000634.

Bonn, C. D., \& Cantlon, J. F. (2017). Spontaneous, modality-general abstraction of a ratio scale. Cognition 169:36-45. doi: 10.1016/j.cognition.2017.07.012.

de Hevia, M. D., Vanderslice, M., \& Spelke, E. S. (2012). Crossdimensional mapping of number, length and brightness by preschool children. PLoS One 7(4):e35530. doi: 10 . 1371/journal.pone.0035530.

Dramkin, D., \& Odic, D. (2020). How children interface number words with perceptual magnitudes. In S. Denison, M. Mack, Y. Xu, \& B. C. Armstrong (Eds.), Proceedings of the 42nd annual cognitive science society (pp. 32953301). Cognitive Science Society. doi: 10.31234/osf.io/zvmfd.

Ellermeier, W., Kattner, F., \& Raum, A. (2021). Cross-modal commutativity of magnitude productions of loudness and brightness. Attention, Perception, \& Psychophysics, 83, 2955-2967. doi: 10.3758/s13414-021-02324-y.

Fechner, G. T. (1887). Uber die psychischen massprincipien und das Weber'sche Gesetz. Philosophische Studien 4:161-230.

Field, G. D., Sampath, A. P., \& Rieke, F. (2005). Retinal processing near absolute threshold: From behavior to mechanism. Annual Review of Physiology 67:491-514 doi: 10. 1146/annurev.physiol.67.031103.151256.

Jacob, S. N., Vallentin, D., \& Nieder, A. (2012). Relating magnitudes: The brain's code for proportions. Trends in Cognitive Sciences 16(3):157-166. doi: 10.1016/j.tics.2012. 02.002 .

Laurence, S., \& Margolis, E. (2005). Number and natural. The Innate Mind: Structure and Contents 1:216.

Luce, R. D., Steingrimsson, R., \& Narens, L. (2010). Are psychophysical scales of intensities the same or different when stimuli vary on other dimensions? Theory with experiments 
varying loudness and pitch. Psychological Review 117:12471258. doi: $10.1037 / \mathrm{a} 0020174$.

Matthews, P. G., \& Chesney, D. L. (2015). Fractions as percepts? Exploring cross-format distance effects for fractional magnitudes. Cognitive Psychology 78:28-56. doi: 10.1016/ j.cogpsych.2015.01.006.

Odic, D. (2018). Children's intuitive sense of number develops independently of their perception of area, density, length, and time. Developmental Science 21(2):e12533. doi: 10.1111/desc. 12533 .

Piazza, M., Izard, V., Pinel, P., Le Bihan, D., \& Dehaene, S. (2004). Tuning curves for approximate numerosity in the human intraparietal sulcus. Neuron 44(3):547-555. doi: 10.1016/j.neuron.2004.10.014.

Pica, P., Lemer, C., Izard, V., \& Dehaene, S. (2004). Exact and approximate arithmetic in an Amazonian indigene group. Science (New York, N.Y.) 306(5695):499-503. doi: 10. 1126/science.1102085.

Rieke, F., \& Baylor, D. A. (1998). Single-photon detection by rod cells of the retina. Reviews of Modern Physics 70(3):1027. doi: 10.1103/RevModPhys.70.1027.

Stevens, S. S. (1957). On the psychophysical law. Psychological Review 64(3):153-181. doi: 10.1037/h0046162.

Stevens, S. S. (1961). To honor Fechner and repeal his law. Science (New York, N.Y.) 133:80-86.

doi: 10.1126/science.133.3446.80. 